\title{
Optimal micromanipulator design for gathering magnetic beads
}

\author{
Shunsuke Tanaka, Ryosuke Ichikawa, Yusuke Yodo, Masahiro Akiyama \\ ${ }^{1}$ NIT Nagano College, 716 Tokuma Nagano-shi, Nagano 381-8550, Japan \\ ${ }^{2}$ Chiba University, 1-33 Yayoi-cho Inage-ku Chiba-shi, Chiba 263-8522, Japan \\ *akiyama@nagano-nct.ac.jp
}

\begin{abstract}
1. Abstract
In this study, we evaluated the optimal geometry for collecting magnetic beads. As a result of making a square shaped coil in the previous research, it was confirmed that magnetic beads were collected at the center and the corner. However, this result was not satisfactory. Because we did not intend for magnetic beads to be collected at the corner of the coil. Therefore, in order to evaluate the shape of the optimal layout, we made and evaluated round, square, and triangular coils, respectively. As a result, it was confirmed that the round coil had the property of collecting magnetic beads in the center more efficiently than the other coils.
\end{abstract}

\section{Introduction}

Figure 1 shows an image of magnetic beads. Magnetic beads are widely used in the collection and detection of biomolecules such as DNA, proteins, viruses, and cells, as well as in drug delivery systems for transporting drugs to affected areas and in hyperthermia for cancer [1]. Furthermore, fluorescent labeling of fluorescent magnetic beads makes it possible to construct multiplexed detection systems with optical detection [2].

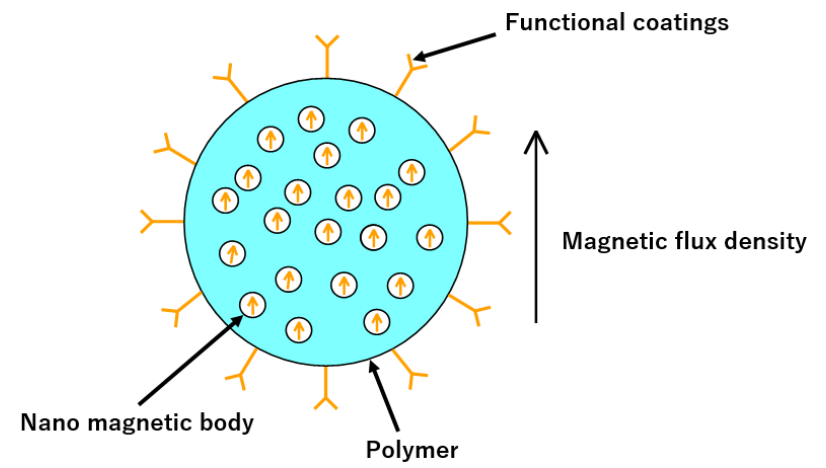

Fig. 1. Image of magnetic beads.

Recently, the development of high-speed and high-sensitivity immunoassay systems that combine a magnetic collection of fluorescent magnetic beads and fluorescence measurement has been required in the medical field [3]. However, the ELISA method currently used for immunoassay using fluorescent magnetic beads is large and expensive [4]. In our laboratory, we have developed a filter-less spectrometer using a compact and inexpensive integrated circuit by installing a sensor for fluorescence detection and a manipulator for magnetic bead collection on an IC chip (Lab on a chip) $[5,6]$. Figure 2 shows an image of a photodiode with magnetic manipulator. The manipulator works to gather the beads for efficiently sensing. In the previous study, it was already confirmed that the magnetic beads were collected at the center of the coil by the coil, but the beads dropped at the corner of the coil did not collect at the center of the coil and stayed there [6].

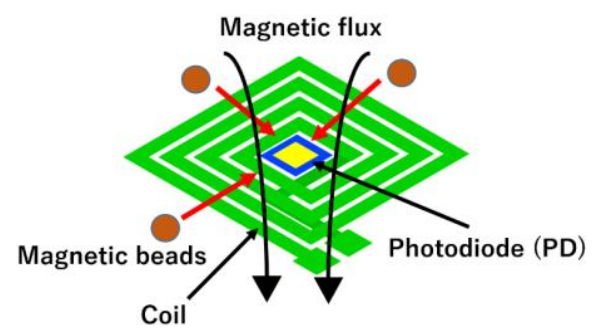

Fig.2. Image of a PD with magnetic manipulator.

In this study, we evaluated the optimal shape for magnetic beads to be collected. By doing so, we thought it would be possible to collect and detect the object to be measured with a single chip. In this paper, we evaluate and report the optimal manipulator geometry for collecting magnetic beads.

\section{Purpose}

The purpose of this study is to fabricate and evaluate a manipulator with an optimal shape for collecting magnetic beads. A coil is used to collect the beads, and by considering the shape of the coil, the beads can be efficiently collected at the intended location. In order to achieve the above objectives, this study evaluates the comparison of the collection characteristics of the corner of the round, square and triangular coils. 


\section{Layout}

Figure 3 shows the overall layout of the IC chip with the magnetic manipulator that is using a $0.8 \mu \mathrm{m}$ one-poly-three-metal (1P3M) CMOS design rule. The chip size is $1.8 \mathrm{~mm} \times 1.8 \mathrm{~mm}$. There are three type layout that are triangle, square and round shape. Figure 4 shows triangle shape coil layout that the size and corner degree are $150 \mu \mathrm{m}$ and $60^{\circ}$. The light blue and green colors are $2^{\text {nd }}$ metal and $3^{\text {rd }}$ metal layer respectively. Figure 5 shows square shape coil layout that the size and corner degree are $96 \mu \mathrm{m}$ and $90^{\circ}$. Figure 6 shows round shape coil layout that size is $96 \mu \mathrm{m}$. These layer width, pitch and coil turn number are $6 \mu \mathrm{m}, 2 \mu \mathrm{m}$ and 5 respectively.

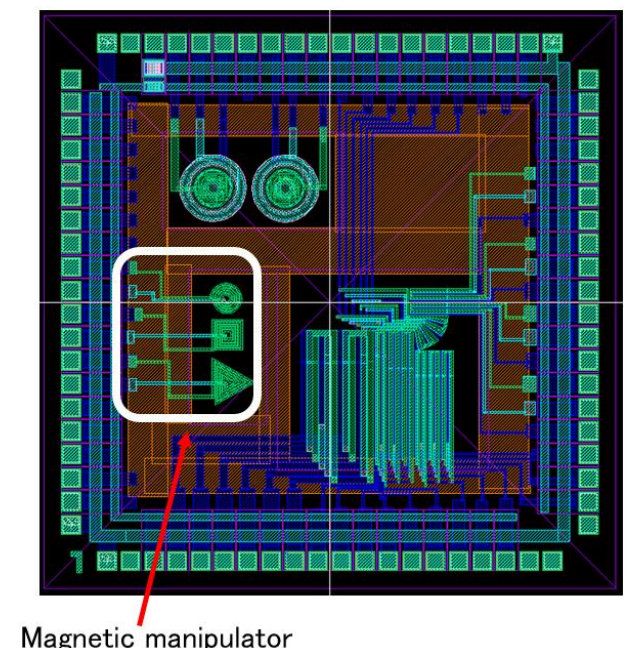

Fig. 3. Layout of the IC chip with the magnetic manipulator.

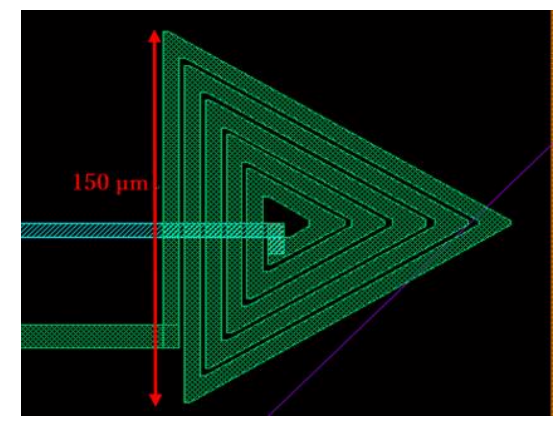

Fig. 4. Triangle shape coil.

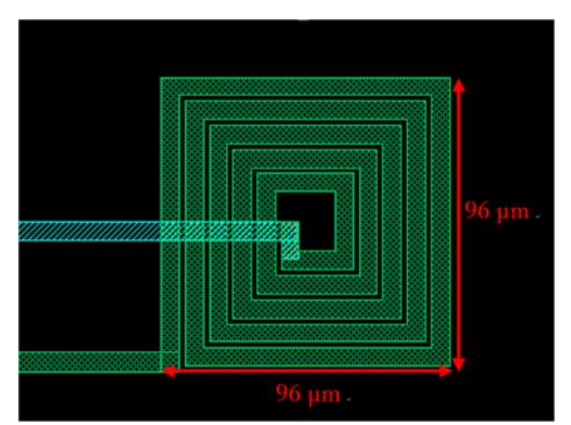

Fig. 5. Square shape coil.

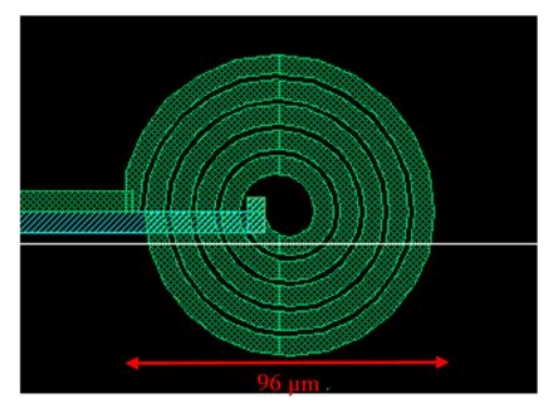

Fig. 6. Round shape coil.

\section{Simulation}

Each magnetic flux density simulated by electromagnetic field analysis software (JMAG). The magnetic flux density distributions generated on the surface of each coil were analyzed and compared when a current of $1 \mathrm{~mA}$ was applied to the coils. The size of each coil is the same as the one shown in the layout. Figure 7 shows the magnetic flux density distribution on triangle shape coil surface. The magnetic flux density is small in the blue area of the figure, and becomes larger as it becomes red. The corner areas have high magnetic flux density compare with straight areas. Figure 8 shows the density distribution characteristics on straight area (A to B) and corner area (A' to B'). The corner areas have high magnetic flux density compare with straight areas. The gap $\left(\Delta \mathrm{B}_{\mathrm{T}}\right)$ of $3^{\text {rd }}$ peak between corner and straight is $0.07 \mathrm{mT}$.

Magnetic flux density $B[T]$

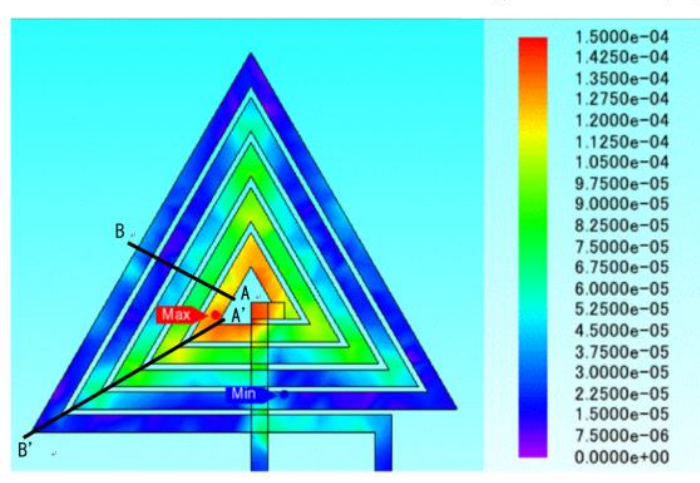

Fig. 7. Magnetic flux density distribution generated in a triangle shape coil.

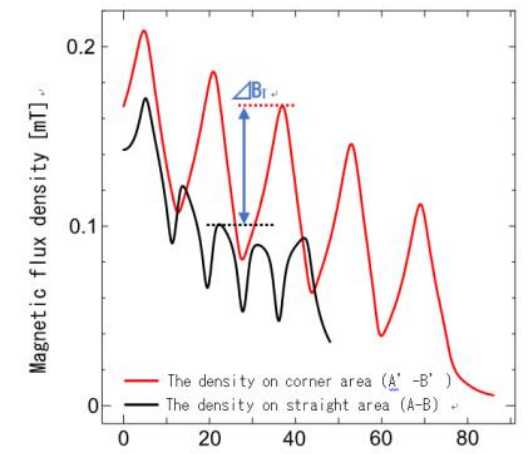

Distance on corner area $\left(A^{\prime}-B^{\prime}\right)$ and straight area $(A-B) \quad[\mu m]$

Fig. 8. Magnetic flux density distribution on corner area(A'-B') and straight area(A-B) in a triangle shape coil 
Figure 9 shows the magnetic flux density distribution on square shape coil surface. The corner areas have high magnetic flux density compare with straight areas. Figure 10 shows the density distribution characteristics on straight area (A to B) and corner area (A' to B'). The corner areas have high magnetic flux density compare with straight areas. The gap $\left(\Delta \mathrm{B}_{\mathrm{S}}\right)$ of $3^{\text {rd }}$ peak between corner and straight is $0.05 \mathrm{mT}$.

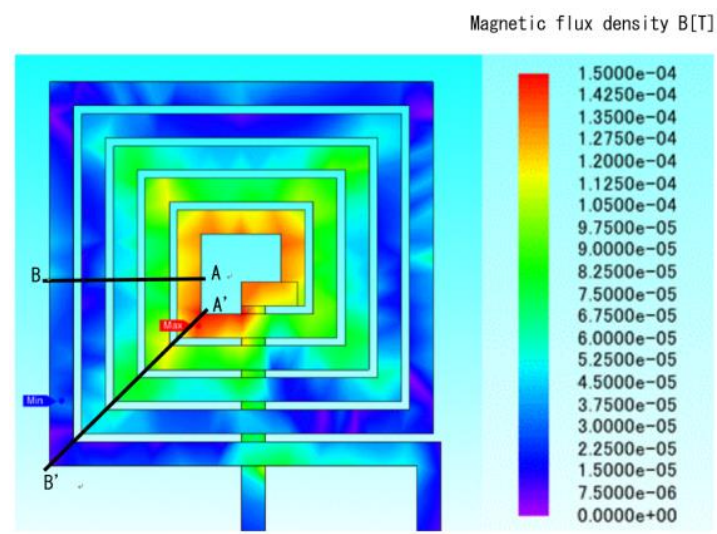

Fig. 9. Magnetic flux density distribution generated in a square shape coil.

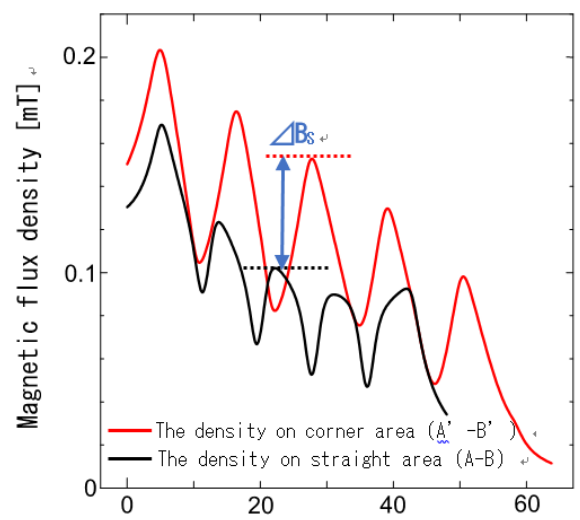

Distance on corner area ( $\left.A^{\prime}-B^{\prime}\right)$ and straight area (A-B) [ [ m ]

Fig. 10. Magnetic flux density distribution on corner area(A'B') and straight area(A-B) in a square shape coil.

Magnetic flux density $\mathrm{B}[\mathrm{T}]$

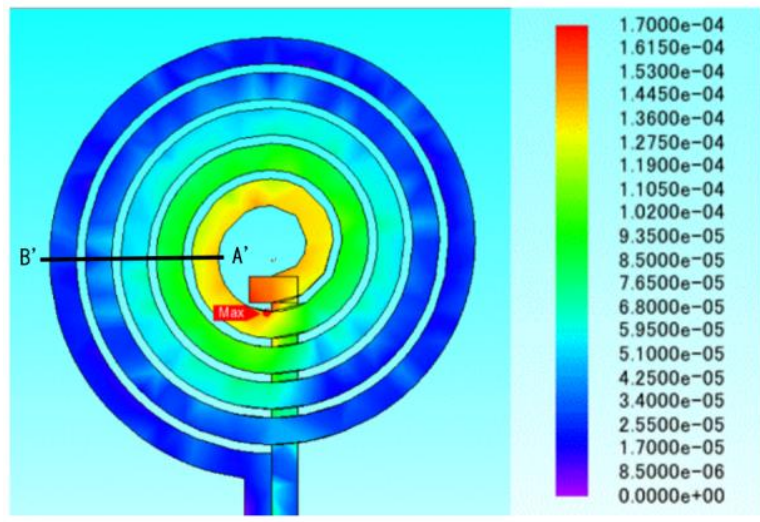

Fig. 11. Magnetic flux density distribution generated in a round shape coil.
Figure 11 shows the magnetic flux density distribution on round shape coil surface. The magnetic flux density distribution is uniform in the round coil because there are no corner portions. In comparison, the square and triangular coils in Figs. 7 and 9 have a stronger magnetic flux density distribution at the corners than the straight parts. Therefore, it is thought that magnetic beads can be collected efficiently by using round-shaped coils in actual measurement.

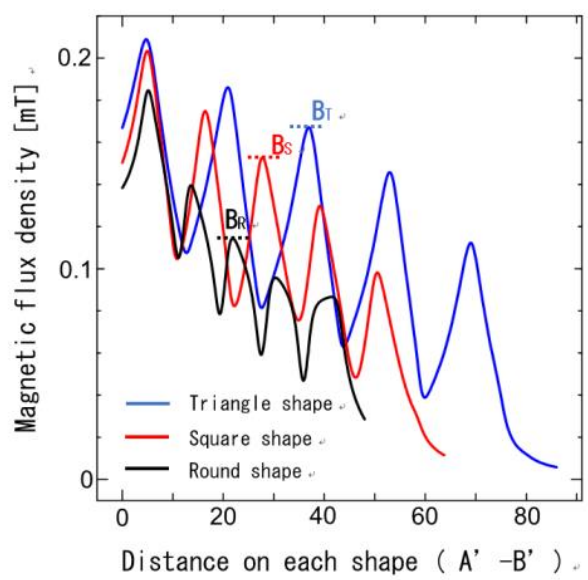

Fig. 12. Magnetic flux density distribution characteristics on the distance (A'-B') in three type shapes.

Figure 12 shows the density distribution characteristics on the distance (A' to B') in three type shapes. The density of $3^{\text {rd }}$ peak values of triangle $\left(\mathrm{B}_{\mathrm{T}}\right)$, square $\left(\mathrm{B}_{\mathrm{S}}\right)$ and round shapes $\left(\mathrm{B}_{\mathrm{R}}\right)$ are 0.17 $\mathrm{mT}, 0.15 \mathrm{mT}$ and $0.115 \mathrm{mT}$ respectively. The $B_{R}$ is small compare with other shapes but it is uniform on the same distance.

\section{Experiment}

Figure 13 shows the fabricated IC chip with the dam that is using a $0.8 \mu \mathrm{m}$ one-poly-three-metal (1P3M) CMOS design process. The dam depth, area and volume are $500 \mu \mathrm{m}, 1.4 \mathrm{~mm} \times 1.4 \mathrm{~mm}$ and $0.98 \mathrm{~mm}^{3}$ respectively.

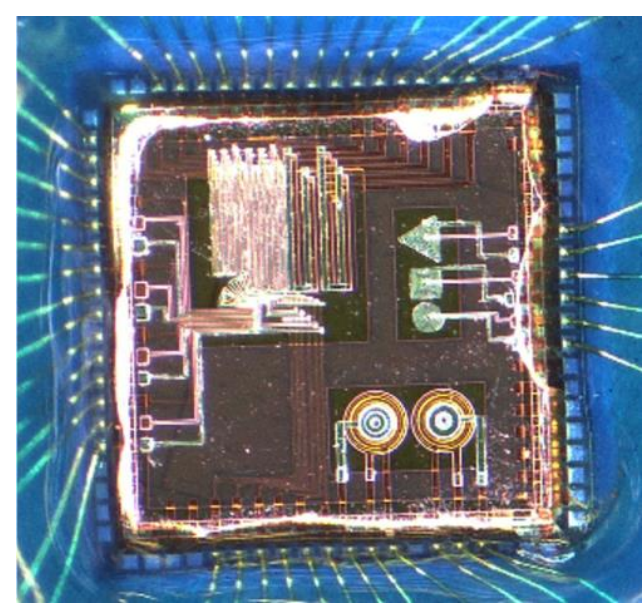

Fig. 13 Fabricated IC chip with the dam. 
Figure 14 shows the measurement circuit for a magnetic beads collection. In this circuit, a current of $12 \mathrm{~mA}$ was supplied from a DC power supply. The magnetic beads dropped on the coil were observed using an OLYMPUS polarized light microscope with a dedicated PC application.

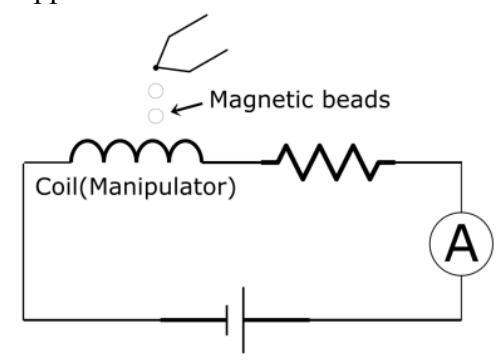

Fig. 14. Measurement circuit for a magnetic beads collection.

"Dynabeads protein A" was adopted as the magnetic beads used in this experiment. These magnetic beads have a particle size of $2.6 \mu \mathrm{m}$ and a weight of about $1.1 \times 10^{-8} \mathrm{mg} /$ particle. The beads were suspended in phosphate-buffered saline (PBS) and diluted to about 15 times with pure water to make the concentration easy to observe with a microscope.

\section{Results and Discussion}

\subsection{Collection characteristics of angled coil}

Figure. 15 and 16 show the triangle and square shape coils with magnetic beads. Before applying current, the beads are not moving $(\mathrm{T}=0 \mathrm{sec})$ and the beads are dispersed on IC surface. When applying current, the beads move to center. After $300 \mathrm{sec}$, the beads is gathering to center and shows the line of the magnetic fields.

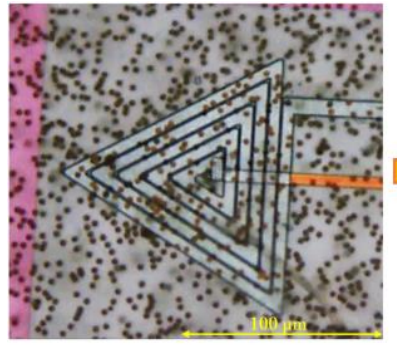

$\mathrm{T}=0 \mathrm{~s}$

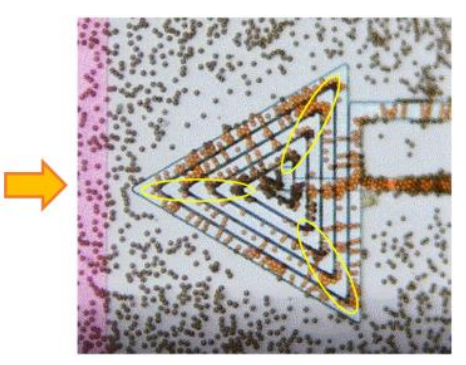

$\mathrm{T}=300 \mathrm{~s}$
Fig. 15. Triangle shape coil with magnetic beads.

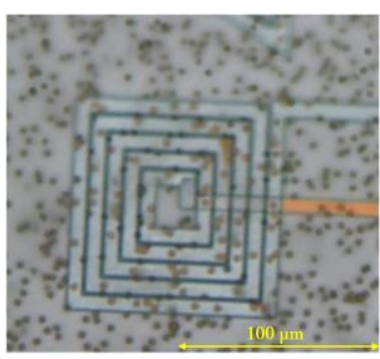

$\mathrm{T}=0 \mathrm{~s}$

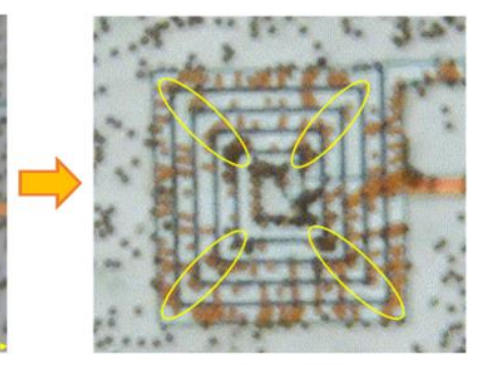

$\mathrm{T}=300 \mathrm{~s}$
Fig. 16. Square shape coil with magnetic beads.
In both Fig. 15 and Fig. 16, magnetic beads are gathered in the center of the coil, but many magnetic beads are also gathered in the corner surrounded by the yellow oval.

\subsection{Collection characteristics of round coil}

Figure 17 shows the round shape coil with magnetic beads.

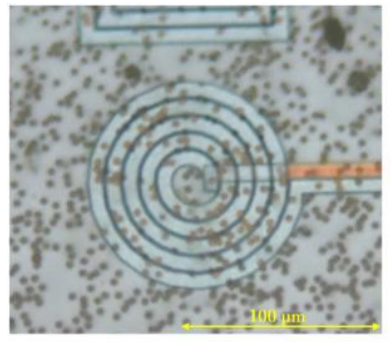

$\mathrm{T}=0 \mathrm{~s}$

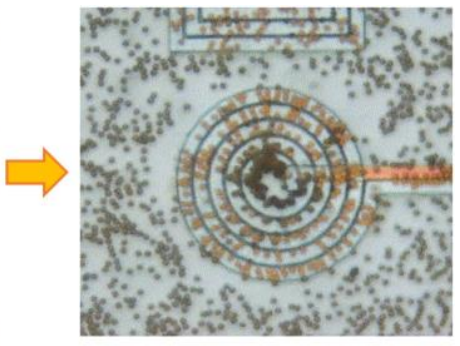

$\mathrm{T}=300 \mathrm{~s}$
Fig. 17. Round shape coil with magnetic beads.

By eliminating the corners, the magnetic beads moved toward the center of the coil from all directions, rather than gathering locally. In addition, the number of beads decreased at distances up to about $10 \mu \mathrm{m}$ from the edge of the coil. In this experiment, it took about 300 seconds for the magnetic beads to be induced to the center of the coil. Therefore, we can see that round-shaped coil can efficiently collect magnetic beads in the center of the coil.

\section{Conclusions}

The purpose of this study is to evaluate the optimal geometry for collecting magnetic beads. Therefore, we fabricated round, square, and triangular coils on the IC chip. From the simulation results, it was confirmed that the magnetic flux density of the round coil was concentrated in the center of the coil compared to the coil with corners. Also, from the actual measurement results, by eliminating the corners of the coil and making it round, the magnetic beads gathered efficiently in the center of the coil. Therefore, the round shape was found to be the best shape.

\section{References}

(1) T. Matsunaga, T. Yoshino "Application of Bio-Nano Magnetic Beads to Medical Care", Special feature: Surface treatment in the medical field, Vol.59, No6, pp.377 - 381 (2008)

(2) J. Sharma, S. Ishizawa, R. Yukino, T. Takamura, N. Hanyu, H. Yasuno, H. Handa, A. Sandhu "Fast and sensitive medical diagnostic protocol based on integrating circular current lines for magnetic washing and optical detection of fluorescent magnetic 
nanobeads", Sensing and Bio-Sensing Research, Vol.9, pp.7-12 (2016)

(3) H. Takehara, M. Nagasaki, K. Sasagawa, T. Noda, T. Tokuda, H. Noji, J. Ohta "A lensless digital enzymelinked immunosorbent assay (ELISA) system using a CMOS image sensor for fluorescence detection" The Institute of Image Information and Television Engineers Technical report, Vol.39, No.16, pp.33-35 (2015)

(4) Alexander van Reenen, Arthur M. de Jong, Jaap M. J. den Toonder, Menno W. J. Prins "Integrated lab-on-chip biosensing systems based on magnetic particle actuation - a comprehensive review", Lab Chip, Vol.14, pp.1966 1986 (2014)

(5) M. Akiyama, K. Miyazawa and K. Sawada, "High sensitive filter-less fluorescence detection method using an avalanche photodiode", Journal of Energy and Power Engineering, Vol. 10, No. 4, pp.268-273, (2017).

(6) R. Ichikwa, S. Tanaka, M. Akiyama, Y. Yodo, "Gathering characteristics of micro magnetic beads using a micro manipulator for fluorescence sensor", The Institute of Erectrical Engneers of Japan (IEEJ), pp.117, (2021) 